\section{P-008 RADIOGRAPHIC AND CLINICAL OUTCOME ANALYSIS OF MONO-PLANE VERSUS BI-PLANE ANGIOGRAPHY SUITES IN STROKE THROMBECTOMIES}

M Nouri ${ }^{*}$, J Lee, J Mocco, P Singh. Mount Sinai Hospital, New York, NY

\subsection{6/neurintsurg-2019-SNIS.44}

Introduction With emerging evidence in recent years proving efficacy of endovascular thrombectomy in treatment of acute strokes, there are a growing number of Primary Stroke Centers in the United States launching interventional neuroradiology services to perform acute thrombectomies at their own institutions rather than transferring patients to a higher level of stroke care. Efficacy and speed of recanalization in these hospitals that may not have biplane angiography available could be brought into question. In our current health system we have a team of multiple interventionalists performing thrombectomies in five hospitals with either mono-plane or biplane angiography suites. In this study we aimed to compare the angiographic and clinical outcome of acute stroke patients who underwent thrombectomy in bi-plane angio suites with those of performed in mono-plane systems.

Methods In this retrospective analysis, 198 patients with isolated middle cerebral artery occlusions who underwent acute intervention from March 2015 to August 2018 were included. Procedures were performed at five different hospitals in our health system. Three of these hospitals use mono-plane rooms and two centers use bi-plane machines. The same group of Neurointerventionlists performed thrombectomies at all hospitals.

Results A total of 198 patients with MCA occlusions were included with $160(80.8 \%)$ cases performed in bi-plane angio suites. Recanalization time was 9.9 minutes longer in monoplane group (66.9 \pm 56.0 vs. $56.8 \pm 45.6$ minutes) but this did not reach statistical significance $(\mathrm{p}>0.05)$. Bi-plane angiography was not significantly associated with higher recanalization rates (TICI $2 \mathrm{~B} / 3 \quad 94.2 \%$ vs. 91.4\%, p >0.05). With respect to clinical outcomes, there was no significant difference between bi-plane angiography vs. mono-plane in the National Institute of Health Stroke Scale at 90 days $(20$ vs. $21, \mathrm{p}>0.05)$. There was no significant difference in a favorable modified Rankin Scale score (mRS $\leq 3$ ) at 90-days between the bi-plane and mono-plane cohorts $(51.7 \%$ vs. $47.7 \%, \mathrm{p}<0.05)$.

Conclusion Although performing stroke thrombectomy with monoplane angiography could theoretically delay recanalization compared to the use of a bi-plane machine, we found no significant difference between these groups in favorable radiographic reperfusion (TICI $2 \mathrm{~B} / 3$ ) or favorable clinical outcomes $(\mathrm{mRS} \leq 3)$ at 90 -day follow-up in our cohort of patients with MCA occlusions. This data not only supports both feasibility and efficacy of performing stroke thrombectomy on monoplane angiography, but it also has implications for increasing access to thrombectomy to more stroke patients without sacrificing the time needed for potentially lengthy hospital transfers.

Disclosures M. Nouri: None. J. Lee: None. J. Mocco: None. P. Singh: None.

\section{P-009 EMBOTRAP EXTRACTION AND CLOT EVALUATION AND LESION EVALUATION FOR NEUROTHROMBECTOMY: METHODS FOR THE EXCELLENT REGISTRY}

${ }^{1} \mathrm{~A}$ Siddiqui ${ }^{*},{ }^{2} \mathrm{~T}$ Andersson, ${ }^{3} \mathrm{~S}$ De Meyer, ${ }^{4} \mathrm{~K}$ Doyle, ${ }^{5} \mathrm{~J}$ Fiehler, ${ }^{6} \mathrm{~W}$ Hacke, ${ }^{7} \mathrm{R}$ Hanel, ${ }^{8} \mathrm{~T}$ Jovin, ${ }^{9} \mathrm{D}$ Kallmes, ${ }^{10} \mathrm{D}$ Liebeskind, ${ }^{11} \mathrm{~A}$ Yoo, ${ }^{12} \mathrm{O}$ Zaidat, ${ }^{13} \mathrm{R}$ Nogueria. ${ }^{1}$ Neurosurgery, State University of New York at Buffalo, Buffalo, NY; ${ }^{2}$ Neuroradiology, Karolinska Institute, Stockholm, Sweden; ${ }^{3}$ Laboratory for Thrombosis Research, KU Leuven, Campus Kulak Kortrijk, Kortrijk, Belgium; ${ }^{4}$ Physiology, National University of Ireland, Galway, Galway, Ireland; ${ }^{5}$ Neurosurgery, University Medical Center Hamburg, Hamburg, Germany; ${ }^{6}$ Neurology, University of Heidelberg, Heidelberg, Germany; ${ }^{7}$ Neurosurgery, Baptist Stroke and Cerebrovascular Center, Jacksonville, FL; ${ }^{8}$ Neurology, University of Pittsburgh Medical Center, Pittsburgh, PA; ${ }^{9}$ Radiology, Mayo Clinic, Rochester, MN; ${ }^{10}$ Neurology, University of California Los Angeles, Los Angeles, CA; ${ }^{11}$ Interventional Radiology, Texas Stroke Institute Dallas-Fort Worth, Buffalo, NY; ${ }^{12}$ Mercy St Vincent Medical Center, Toledo, OH;

${ }^{13}$ Neurosurgery, Emory University School of Medicine, Atlanta, GA

10.1136/neurintsurg-2019-SNIS.45

Rationale The EmboTrap Revascularization Device has been shown to be effective in achieving successful reperfusion in emergent large vessel occlusion (ELVO) patients, with clinical outcomes comparable to existing stent retriever devices. Prospective multicenter data on the real-world experience with EmboTrap is not available.

Aim The primary objective of the study is to assess the efficacy of routine, real-world experience of the EmboTrap and to explore the associations between imaging characteristics at presentation, including volume of core and penumbra, final infarct volume, histologic clot characteristics, subject comorbidities, revascularization rate, and clinical outcomes.

Design This is a prospective, global, multi-center, single-arm, observational registry. Up to 1000 subjects at 50 different sites in Europe and US may be enrolled in the study.

Adult patients ( $>18$ years) with angiographically confirmed ELVO, and treated with EmboTrap as first attempted device will be eligible for participation in the study. Patients with confirmed pregnancy or enrolled in another investigational clinical trial will be excluded.

This study will utilize expanded thrombolysis in cerebral infarction score (eTICI) and an independent Core Imaging Laboratory to assess baseline CT/MR imaging (e.g. ASPECTS, infarct volume, clot location, perfusion), procedural angiography (eTICI score for every pass, emboli to new territories, collateral score) and post-procedure CT/MR imaging (infarct volume, intracranial hemorrhage). All imaging will be read independently by an experienced Imaging Core Lab blinded to all clinical data. In addition, an independent Core Laboratory will also perform clot analysis.

Study outcomes Pre-specified endpoints of the study include successful revascularization (modified thrombolysis in cerebral infarction score $\geq 2 \mathrm{~b}$ ), first pass effect (eTICI $\geq 2 \mathrm{c}$ in a single pass without rescue), number of passes, functional independence assessed as modified Rankin Score of $\leq 2$ at 90 days, 2 rates of embolization to new territory, symptomatic intracranial hemorrhage at 24 hours and all-cause mortality at 90 days.

Analysis Standard descriptive statistical summaries will be provided. For categorical endpoints, two-sided exact 95\% confidence intervals will be presented. Regression analyses will be 